\title{
Effective Interactions Between Rigid Polyelectrolytes and Like-charged Planar Surfaces
}

\author{
R. Jay Mashl* and Niels Grønbech-Jensen ${ }^{\dagger}$ \\ Theoretical Division and the Center for Nonlinear Studies, MS B258, \\ Los Alamos National Laboratory, Los Alamos, NM 87545 \\ (Final version published in The Journal of Chemical Physics, 109, 4617-4623 (1998))
}

\begin{abstract}
We study the effective interaction between a planar array of uniformly negatively charged, stiff rods parallel to a negatively charged planar substrate in the absence of salt in a continuous, isotropic dielectric medium. Using Brownian dynamics simulations, we examine the general effects of counterion valence, rod spacing, macroion charge densities, and the rod size on the attractive rod-surface interaction force. At room temperature divalent as well as monovalent counterions mediate an interaction that can be repulsive or attractive upon adjusting either the macroion charge densities or the rod radius. Finally, we examine the effects of discretizing the surface charge as laterally mobile monovalent anions and of electrostatic images in the substrate.
\end{abstract}

*Present address: NCSA, University of Illinois, 405 N. Mathews, Urbana, IL 61801; mashl@ncsa.uiuc.edu

${ }^{\dagger}$ ngj@lanl.gov 


\section{INTRODUCTION}

In the past few decades many investigations have provided evidence that effective attractions can occur between like-charged macroions. Attraction between like-charged surfaces or rod-like macroions has been observed numericallyt 10 mainly through the use of Monte Carlo methods, 19 but also through Brownian dynamics simulations.10_Most of these studies have featured ions as mediating the attraction, but a few studies 11 have found an attraction by the use of polyelectrolytes of the opposite charge. Attraction has also beep-seen analytically 122 through 31 arious methods, including integral-equation approaches, 13 -26 density-functional theories, 27,28 and lattice models.29., 30 Finally, a variety of experiment\$22 3243 has indicated attractions as well. For example, X-ray scattering studies on colloidal particles made of poly(styrene sulfonate) 3435 or silica 36 have shown phase separation behavior, and when coupledwith externally applied osmotic stress, has shown DNA aggregation by multivalent ions $37-38$ and ligands.37 Effective diffusion constants derived from light scattering measurements on salt-free systems of poly(styrene sulfonate) of varying molecular weights 39 or of varying concentration 40 have suggested macroion bundling. Light scattering combined with electron microscopy has shown the reversible aggregation behavior of various filamentous biopolymers in the presence of multivalent metal ions or peptide homopolymers. 41 Deuterium-NMR and ${ }^{23} \mathrm{Na}$-NMR have been used to derive phase diagrams of lamellar liquid crystalline systems. 12 Finally, in calcium clays the attraftive forces between the mica surfaces have been measured using the surface force apparatus, 22 and the effects of osmolyte concentrations on the clays have been studied by X-ray scattering. 13 Thus, there is substantial evidence of effective attractions between charged objects of the same sign.

Theory has shown that ion-ion correlations (i.e., correlations in the fluctuations of the ion positions) are the source of the attractions in the numerically and analytically studied model systems. Mean-field descriptions, such as the Debye-Hückel and Poission-Boltzmann equations, 44 which are often used to calculate forces between macroions, 45 4 48 always fail to yield this attraction because they do not account for interparticle correlations 49 On the other hand, integral equation approaches naturally possess the framework for including such interactions. Fluctuation theories also can yield an attraction. Oosawa 12 has shown (to dipole order) that the coupling of longitudinal fluctuations in the counterionic atmospheres along two parallel, like-charged, rod-like macroions produces an electrostatic attraction. Whereas Oosawa has predicted this attraction to increase with temperature, we recently found by the use of Brownian dynamics simulations that it increases as the temperature decreases. $\frac{10}{0}$

Since the effective interaction between like-charged surfaces or two like-charged rods has been fairly well studied, we will address here the effective interaction between rod-like macroions and a like-charged surface. The geometry is motivated by experimental systems involving highly negatively charged DNA molecules and negatively charored membranes. In fact, experimental methods have been developed (for reviews, see Ref.5051) to adsorb DNA onto negatively charged substrates. Among these methods is the adhesion of DNA to mica surfaces by the use of multivalent cation $\$ 22$ or cationic detergents.62 The treatment of mica surfaces with a solution of magnesium acetate, calcium acetate, or magnesium chloride has been seen to stabilize adsorbed DNA and subsequently give reproducible imaging by atomic force microscopy 2258 or scanning tunneling microscopy 5961 These treatments are believed to displace potassium ions in the mica with divalent cations so as to provide stronger binding sites. Similarly, divalent cations have been shown to be more successf than monovalent cations in binding DNA to quartz sand in DNA-degradation studies.63 Studies on gene-delivery systems have reported on the adsorption of DNA to anionic liposomes with 64 and without 65 the use of positively charged binding proteins, rather than simple cations. Finally, two-dimensional, fingerprint-like films of DNA strands have been found to adhere to cationic bilayers deposited onto untreated mica substrates.66.67 Thus, experiments reveal that adsorption of rod-like macroions onto like-charged substrates can be mediated by oppositely charged ions, proteins, or membranes.

The model system we will investigate, as suggested the experiments above, is an array 
of rigid, negatively charged rods parallel to a negatively charged surface. The question on which we focus is, can a charged surface attract a like-charged rod? Although the DNA in the above experiments is admittedly flexible and the surfaces are not necessarily strictly planar, we investigate this simpler system in order to gain insight into the more complicated systems. For simplicity, we omit salt from our study in order to examine the fundamental nature of the interaction between the like-charged macroions. Our method is to fix the array of rods at certain distances from the surface, compute the time-averaged force on the rods perpendicular to the surface using Brownian dynamics simulations, and investigate the dependence of this force on a systematic variation of model parameters, such as array height, macroion charge densities, rod spacing, counterion valence, and rod size. We also examine the roles of electrostatic images and the discretization of the surface charge into mobile monovalent anions. In the next section we present the pairwise potential energy functions used in the simulations. These potentials model only the classical electrostatic interactions and neither distinguish between counterion species of the same valence nor consider solvent structure. We then summarize the Brownian dynamics algorithm by which the representations of the counterions are moved by deterministic and thermal forces and follow with the results and a discussion.

\section{THEORY}

\section{A. The Model}

The system we consider is an isotropic, continuous dielectric medium containing negatively charged rods and their counterions above a supported bilayer of anionic lipids. Our simulational unit cell model (Fig. 1) is a rectangular open half-space $(z>0)$ of dielectric constant $\epsilon_{1}\left(\approx 80\right.$ for an aqueous medium) with dimensions $L_{x}$ and $L_{y}$ that contains a single line charge of uniform charge density $\lambda$ located a distance $d$ from a fixed, flat surface of a specified average charge density $\sigma$. This surface density can be either a uniform density distribution or a collection of discrete, mobile monovalent anions, representing, e.g., a fluid-phase mixture of charged and neutral lipids. The unit cell is replicated the $x$ and $y$ directions to produce an infinite array of infinitely long, parallel line charges with a spacing $L_{x}$ and with a repeat distance $L_{y}$ in the $y$ direction. There is no periodicity in the $z$ direction. This replicated dielectric medium is bounded below $(z \leq 0)$ by an isotropic, continuous half-space of dielectric constant $\epsilon_{2}(\approx 3)$ representing the dielectric layer formed by the hydrocarbon tails of the lipids. The dielectric discontinuity at $z=0$ produces electrostatic images in the substrate in - rder to satisfy the boundary conditions on the dielectric displacement field at the interface.68 A point particle of charge $q_{i}$ placed in the medium with $\epsilon_{1}$ at a distance $z_{i}$ away from the planar interface will produce an electrostatic image charge of charge $q_{i}^{\prime}=q_{i}\left(\epsilon_{1}-\epsilon_{2}\right) /\left(\epsilon_{1}+\epsilon_{2}\right)$ at the symmetric position $-z_{i}$. An electrostatic image rod is produced similarly. As a result, a uniform surface charge density (or the charge of individual mobile monovalent surface anions) increases by a factor of $2 \epsilon_{1} /\left(\epsilon_{1}+\epsilon_{2}\right)$.

The interaction potentials consist of pairwise, long-ranged electrostatic (Coulombic) forces and short-ranged, non-electrostatic repulsions. We make no approximations regarding the Coulomb interations. For a uniformly charged surface, the ion-surface electrostatic interaction $V_{\mathrm{i}-\mathrm{s}}(z)$ is a function of the distance $z$ the ion with charge $q_{i}$ is from the surface:

$$
V_{\mathrm{i}-\mathrm{s}}(z)=-\frac{q \sigma}{2 \epsilon_{1} \epsilon_{0}} z
$$

where $\epsilon_{0}$ is the permittivity of vacuum. The rod-surface electrostatic contribution $V_{\mathrm{r}-\mathrm{s}}(z)$ per unit cell for a uniform surface charge distribution is similarly 


$$
V_{\mathrm{r}-\mathrm{s}}(z)=-\frac{\lambda L_{y} \sigma}{2 \epsilon_{1} \epsilon_{0}} z .
$$

The ion-ion electrostatic potential energy $V_{\mathrm{i}-\mathrm{i}}(\Delta \mathbf{r})$ for an ion with charge $q_{1}$ at the point $(x+\Delta x, y+\Delta y, z+\Delta z)$ and an ion with charge $q_{2}$ and its replicas located at $\left(x+m L_{x}, y+n L_{y}, z\right)$, where $m, n$ are integers, resulting from the two-dimensional replication of the unit cell in the $x$ and $y$ directions, is given exactly by an absolutely convergent, double summation. The ion-ion potential energy $V_{\mathrm{i}-\mathrm{i}}(\Delta \mathbf{r})$ is 69

$$
\begin{aligned}
& V_{\mathrm{i}-\mathrm{i}}(\Delta \mathbf{r})=\frac{q_{1} q_{2}}{4 \pi \epsilon_{1} \epsilon_{0}}\left\{\frac{4}{L_{x}} \sum_{n=1}^{\infty} \cos \left(2 \pi \frac{\Delta x}{L_{x}} n\right) \sum_{k=-\infty}^{\infty}\right. \\
& K_{0}\left\{2 n \pi n\left[\left(\frac{L_{y}}{L_{x}}\right)^{2}\left(\frac{\Delta y}{L_{y}}+k\right)^{2}+\left(\frac{\Delta z}{L_{x}}\right)^{2}\right]^{1 / 2}\right\} \\
& -\frac{1}{L_{x}} \ln \left[\cosh \left(2 \pi \frac{\Delta z}{L_{y}}\right)-\cos \left(2 \pi \frac{\Delta y}{L_{y}}\right)\right] \\
& \left.-\frac{\ln 2}{L_{x}}\right\} .
\end{aligned}
$$

When the surface consists of discrete ions, the potentials $V_{\mathrm{i}-\mathrm{s}}(z)$ and $V_{\mathrm{r}-\mathrm{s}}(z)$ are identically zero, and all electrostatic interactions with the surface are taken according to Eq. (3). The self-energy $V_{\mathrm{i}-\mathrm{i}}^{(0)}$ that arises from an ion interacting with its own replicas is found by evaluating the expression

$$
V_{\mathrm{i}-\mathrm{i}}^{(0)}=\frac{1}{2} \lim _{r \rightarrow 0}\left(V_{\mathrm{i}-\mathrm{i}}(\Delta \mathbf{r})-\frac{q_{i}^{2}}{4 \pi \epsilon_{1} \epsilon_{0} r}\right)
$$

and is given in Ref 69 The ion-rod potential energy $V_{\mathrm{i}-\mathrm{r}}(\Delta \mathbf{r})$ is the logarithmic interaction between a point particle and a one-dimensional array of line charges. The analytic form of $V_{\mathrm{i}-\mathrm{r}}(\Delta \mathbf{r})$ is derived from the potential energy of an ion interacting logarithmically with a two-dimensional array of line charges arranged on a rectangular lattice (see Eq. (14) in Ref.0 by eliminating one of the dimensions. The result is given by

$$
\begin{aligned}
V_{\mathrm{i}-\mathrm{r}}(\Delta \mathbf{r})= & -\frac{q \lambda}{4 \pi \epsilon_{1} \epsilon_{0}} \ln \left\{2 \left[\cosh \left(2 \pi \frac{\Delta z}{L_{x}}\right)\right.\right. \\
& \left.\left.-\cos \left(2 \pi \frac{\Delta x}{L_{x}}\right)\right]\right\} .
\end{aligned}
$$

The corresponding ion-rod self-energy simplifies similarly from Ref 0 to

$$
V_{\mathrm{i}-\mathrm{r}}^{(0)}=-\frac{q \lambda}{4 \pi \epsilon_{1} \epsilon_{0}} \ln \frac{2 \pi}{L_{x}}
$$

The interaction between the real ions and electrostatic image ions as well as between the real ions (rods) and electrostatic image rods (ions) is obtained directly from the periodic potentials in Eqs. (3-5), respectively, up to a factor of $2 \epsilon_{1} /\left(\epsilon_{1}+\epsilon_{2}\right) \approx 1.93$ ). Thus, interactions involving electrostatic images are treated without approximation. Finally, the ion-rod and ion-surface short-ranged, non-electrostatic repulsion energies were modeled as $A_{\mathrm{i}-\mathrm{r}} / r^{11}$ and 
$A_{\mathrm{i}-\mathrm{s}} / r^{10}$, respectively, to prevent the ions from collapsing electrostatically. The combination of the Coulomb interaction and short-ranged repulsion yields an optimal ion-rod distance, i.e., a rod radius $r_{0}$, which was considered among the model parameters. Values for the coefficients $A_{\mathrm{i}-\mathrm{r}}$ and $A_{\mathrm{i}-\mathrm{s}}$ and the resulting optimal ion-rod and ion-surface distances are given below. The ion-rod short-ranged interaction is taken according to the minimum image convention.

The values of the parameters in our reference system are taken to be reasonably representative of those found in the literature. Under physiological conditions the DNA found in most organisms is 71 in the so-called B-form, a double-helical structure of approximate radius of $12 \AA$, bearing two negatively charged phosphate groups per base pair (i.e., every $3.4 \AA$ on average). Taken as a whole, calcium clays are reported 20 to have areas per unit charge ranging from $51-135 \AA^{2}$, whereas negatively charged lipids known 72 to form (mono/bilayers) have a median surface area per molecule of about $51 \AA^{2}$. On the basis of this latter value alone, we choose our reference system to consist of rods with an equivalent linear charge density $\lambda_{\text {ref }}=-e / 1.7 \AA$, lateral spacing $L_{x}=30 \AA$, and a surface with an average charge density $\sigma_{\text {ref }}=-e / 51 \AA^{2}$. As we were unable to find data on the spacing of DNA in fully two-dimensional films adsorbed directly onto negatively charged substrate, we do not know whether a lateral spacing of $30 \AA$ is reasonable However, DNA molecules adsorbed onto cationic-lipid bilayers supported by mica have67 an interhelical spacing that depends nonmonotonically on the concentration of divalent magnesium cations in solution and lies in the range 35-45 A. Furthermore, DNA molecules in sodium chloride solution are known 3 . 4 to pack into hexagonal arrays with lattice spacings ranging between 25-40 . Dielectric constants in our system are taken to be $\epsilon_{1}=80$ and $\epsilon_{2}=3$ or 80 . In the former case, when electrostatic images are included, an ion or rod always repels its own electrostatic image. All simulations were carried out at $300 \mathrm{~K}$.

Values of $A_{\mathrm{i}-\mathrm{r}}$ of $7.5 \times 10^{7}, 3.27 \times 10^{4}$, and $1.8 \times 10^{1} \mathrm{kcal} \AA^{11} / \mathrm{mol}$ yielded rod radii $r_{0}$ of $5.6,2.8$, and $1.4 \AA$, respectively, in the reference macroion system. Unless otherwise specified, the second $A_{\mathrm{i}-\mathrm{r}}$ value was chosen as the reference rod value regardless of the actual

macroion charge densities. For all simulations $A_{\mathrm{i}-\mathrm{s}}$ was fixed at $6.63 \times 10^{2} \mathrm{kcal} \AA^{10} / \mathrm{mol}$, yielding an optimal ion-surface distance of $2.4 \AA$ for the reference system. The reference value of rod radius $r_{0}=2.8 \AA$ is not equal to the real size of DNA. The reason for this is that the rod size and the correct ion-rod interaction energy cannot be imposed simultaneously within our model. We believe it would be more correct to account for the electrostatic "binding" energy between a counterion and a DNA ion (on the order 75 of a few $k_{\mathrm{B}} T$ ) rather than the actual DNA size.

\section{B. Simulation Method}

The motion of a collection of particles undergoing Brownian dynamicscorresponds to the long-time behavior of the Langevin equation in the overdamped limit. $\mathbb{6}$ The Langevin equation expresses a balance of deterministic and thermal, random forces on each particle at any given moment in time $t$. For each particle $i$ with mass $m_{i}$, position $\mathbf{r}_{i}(t)$, velocity $\mathbf{v}_{i}(t)$, and isotropic friction coefficient $\xi_{i}$, the Langevin equation reads

$$
m_{i} \frac{d \mathbf{v}_{i}(t)}{d t}=-\xi_{i} m_{i} \mathbf{v}_{i}(t)+\mathbf{f}_{i}(\{\mathbf{r}(t)\})+\mathbf{f}_{i}^{*}(t)
$$

where $\mathbf{f}_{i}(\{\mathbf{r}(t)\})$ is the total deterministic force acting on particle $i$ due to the positions $\{\mathbf{r}(t)\}$ of all the particles, and $\mathbf{f}_{i}^{*}(t)$ is the random force acting on particle $i$. This random force is characterized by the fluctuation-dissipation theorem as 


$$
\left\langle\mathbf{f}_{i}^{*}(0) \cdot \mathbf{f}_{j}^{*}(t)\right\rangle=6 m_{i} k_{\mathrm{B}} T \xi_{i} \delta(t) \delta_{i j}
$$

for particles $i$ and $j$, with the time average of the random force over any particle vanishing:

$$
\left\langle\mathbf{f}_{i}^{*}(t)\right\rangle=0 \text {. }
$$

At long times the friction coefficent $\xi_{i}$ is related to the diffusion constant $D$ as $D_{i}=$ $k_{\mathrm{B}} T / m_{i} \xi_{i}$. The inertial term on the left-hand side of Eq. (7) can be set to zero at long times. One discretization of the resulting equation yields an iterative scheme for the time evolution of the system of particles:77.78

$$
\mathbf{r}_{i}(t+\Delta t)=\mathbf{r}_{i}(t)+\frac{\Delta t}{m_{i} \xi_{i}} \mathbf{f}_{i}(\{\mathbf{r}(t)\})+\mathbf{r}_{i}^{*}(\Delta t)
$$

where $\mathbf{r}_{i}^{*}(\Delta t)$ is a random Gaussian displacement with a variance of $2 k_{\mathrm{B}} T \Delta t / m_{i} \xi_{i}$ in each dimension. Given the total potential energy surface for a particle $i, V_{i \text {,tot }}(\{\mathbf{r}(t)\})$, the deterministic force $\mathbf{f}_{i}(\{\mathbf{r}(t)\})$ is given by $\mathbf{f}_{i}(\{\mathbf{r}(t)\})=-\nabla V_{i \text {,tot }}(\{\mathbf{r}(t)\})$.

\section{RESULTS AND DISCUSSION}

Each simulation required the specification of a set of system parameters $\left\{\lambda, \sigma, r_{0}, L_{x}, L_{y}\right\}$, a fixed position of the rods $d$, whether the surface charge density consisted of discrete, mobile monovalent anions, and whether electrostatic images were to be included. The value of $L_{y}$ depended on the particular set of system parameters and fell in the range $17-272 \AA$, thereby giving approximately 80 or more ions in the basic unit cell to help ensure that particle configurations were well sampled. The divalent or monovalent counterions were introduced as a random configuration until the system became overall charge neutral; no additional ions (i.e., co-ions or salt) were added. As the rods were fixed in space, the simulation involved only the motion of the ions due to the explicit pairwise interactions among the ions, the rods, and the single charged surface, as well as the random thermal forces. For the ion motion, the normalized time step was taken to be $\Delta t \leq 0.01$ for $m_{i} \xi_{i} \equiv 1$. During the simulations the vertical and lateral time-averaged forces per unit length on the rod and the vertical time-averaged force per unit $L_{y}$ length on the surface were monitored. After the initial $5-10 \times 10^{4}$ steps were discarded, these averages were accumulated over an additional $2-200 \times 10^{6}$ time steps, depending on the number of simulated particles, system size, macroion charge densities, and counterion valences. Equilibrium was attained when the average force on the $\operatorname{rod}\left\langle F_{z \text {,rod }}\right\rangle$ and surface $\left\langle F_{z \text {,surf }}\right\rangle$ remained constant and the average

lateral force on the $\operatorname{rod}\left\langle F_{z \text {,rod }}\right\rangle \approx 0$. We checked for finite-size effects in $L_{y}$ and found that they do not affect our results significantly.

Numerous simulations with systematic variations of the system parameters were carried out to investigate the general behavior of the system. Figure 2 shows the time-averaged, rod-surface force in the $z$-direction per unit length rod as a function of rod-surface spacing for several rod and uniform surface charge densities for monovalent and divalent counterions, with and without electrostatic images. For the case of monovalent counterions, the top set of two curves shows that the effective rod-surface interaction is always repulsive, whereas the lower set of curves indicates that there are rod-surface distances $d$ for which the interaction is attractive. As can be seen, the interaction becomes more attractive as the charge densities on the rod and surface increase from one to four times the reference charge densities. For the case of divalent counterions, the rod-surface interaction is attractive for half, one times, and double the reference charge densities. The maximal (attractive) force, indicated by the depth of the force-distance profile, increases as the charge densities increase. Divalent counterions are obviously more effective at inducing an attraction than are monovalent counterions. 
The effect of changing the interrod spacing $L_{x}$ on a divalent-counterion system at the reference rod and uniform surface charge densities was examined. For a system containing no electrostatic images, the force-distance profiles for $L_{x}=15,30,60 \AA$ were indistinguishable, and the profiles for systems with electrostatic images were also indistinguishable. Halving (doubling) the rod radius $r_{0}$ also resulted in indistinguishable profiles whose maximal attractive forces, i.e., minima, were deeper (shallower) and located at smaller (larger) $d$ compared to the reference case. Thus, these combinations of $L_{x}$ and $r_{0}$ place these systems essentially in the dilute-rod limit. This dilute-rod regime is a well-defined limit as the effective rod-surface interaction is independent of the initial distribution of counterions. In further decreasing the lateral rod spacing $L_{x}$ (and/or increasing the rod radius) one would eventually encounter a less permeable array of rods. The resulting effective rod-surface force is then expected to depend longer on time and may lead to a significantly different effective rod-surface force as compared to the dilute-rod regime, due to the initial partitioning of counterions between the region exterior to the rods $(z>d)$ and the region between rods and surface $(0<z<d$, in Fig. 1$)$.

Figure 2 can also be interpreted in terms of the equilibrium rod-surface separation distance, $d^{*}$, the point at which force-distance profiles cross zero. In general, for either counterion species, as the macroion charge densities increase, the equilibrium rod-surface distance decreases. This behavior is most striking for monovalent counterions as increasing the macroion charge densities causes $d^{*}$ to move from infinity to a finite distance. Finally, the effect of electrostatic images for both monovalent- and divalent-counterion systems is quite small compared to that which can be achieved through changing only the macroion charge densities.

Changing the macroion charge densities individually leads to a type of phase diagram for the rod-surface force. Figure 3 shows the regions of rod-surface attraction and repulsion for systems of monovalent counterions with uniform charge densities and no electrostatic images. In general, the larger macroion charge densities (on the rod or the surface) lead to an effective attraction, and the lower charge densities lead to repulsion. The parameter set $\lambda / \lambda_{\text {ref }}=\sigma / \sigma_{\text {ref }}=2$ led to only marginal attraction, and this ambiguity is indicated in the figure. The dashed line, as suggested by the data, indicates only a qualitative phase boundary between the attractive and repulsive regions, as the actual shape of the boundary is unknown and would require a more detailed exploration of the macroion density phase space. Doubling the rod radius led to repulsion in each of the nine cases shown in Fig. 3. For a given fixed rod-surface separation distance, an increase in the rod radius tends to obstruct the counterions from entering the region in between the rod and surface, thereby hindering the ions from contributing relevant attractive interactions. Thus, the presence of an attraction bewteen the rods and surface is conditional on not only the macroion charges but also the rod radius.

We examined the effect of macroion charge densities in systems of divalent counterions and found, as in Fig. 1, that an attraction between the rods and surface is evident in systems with macroion uniform charge densities down to $\lambda=0.5 \lambda_{\text {ref }}$ and $\sigma=0.5 \sigma_{\text {ref }}$ with the reference rod size. In these cases, the equilibrium rod-surface distance lies within a quite small range (5-6 $\AA$ ), with the smaller distances corresponding to the higher charge densities. We have not checked whether an attraction exists at even lower charge densities on either macroion.

Finally, the roles of a discrete, mobile surface charge distribution and electrostatic images in systems of monovalent and divalent counterions were examined. Figure 4 shows a comparison of systems with the reference densities $\lambda=\lambda_{\text {ref }}$ and $\sigma=\sigma_{\text {ref }}$ for the cases of 1) a uniformly charged surface with no electrostatic images, 2 ) a uniformly charged surface with electrostatic images, 3) a surface composed of discrete, mobile monovalent anions with no electrostatic images, and 4) same as in 3) but with electrostatic images. The force-distance profiles for the monovalent-counterion systems all show a repulsive rod-surface force and are essentially indistinguishable. Although the divalent force-distance profiles give nearly the same equilibrium rod-surface distances and appear similar in shape, the depths of the pro- 
files vary significantly. The maximal attractive force on the rods is greater for systems with surfaces composed of discrete, mobile charges than for systems with a uniformly charged surface. It is reasonable to attribute this result of enhanced attractions to the increased coupling between the mobile surface ions and the rod and other ions. A surface composed of a mobile charge distribution provides the system with more configurational degrees of freedom, which is, in general, expected to lead to an overall lowering of the free energy of the system. Interestingly, the four force profiles for the divalent-counterion systems all cross near $7 \AA$. We do not know whether this common point is a general phenomenon or an artifact due to our particular system geometry and parameters.

\section{CONCLUSIONS}

In summary, we have presented a systematic study of the effective interactions between a single charged surface and a parallel array of same-sign charged rods in the presence of their counterions with no co-ions (salt). We indeed find an effective attraction between the rods and surface that is mediated by either divalent or monovalent counterions at room temperature in the dilute-rod limit. This attraction results from counterion correlations that overcome the inherent repulsion between the two macroions. Divalent counterions readily mediate this attraction at macroion charge densities that are comparable to those found in highly charged biopolymers and surfaces. Clearly, counterions of higher valence are more effective at inducing attraction between charged macroions of the same-sign charge density, and this trend has been observed experimentally 3,41 and numerically. 10 Although the very high macroion charge densities needed by systems of only monovalent counterions to display an effective attraction are seemingly unphysical, there indeed appears to be polymers with rather high equivalent linear charge densities. One group $\mathbf{E}^{\mathbf{9}}$ has pointed out that the anionic polymers dextran sulfate and heparin have a 2.0-fold greater charge density than DNA. The simulations have further revealed that the higher the macroion charge densities, the smaller the rod-surface equilibrium distance.

We have demonstrated for this rod-surface system that the volume excluded by the rod to the counterions is an important factor in determining macroion attraction or repulsion, particularly for systems of monovalent counterions. An increase in rod radius tends to lower the attraction between the macroions because the average number of counterions in between the macroions is reduced. These results are consistent with our recent work 10 on systems of two identical rods in the absence of salt. In the present paper we also have discussed that the decrease in the maximal attraction due to an increased rod size can be partially offset by an increase in the rod-surface distance, while leading to a new decreased maximal attractive force.

The accounting for electrostatic images generally introduces quite small, repulsive additions to the rod-surface interaction. Whereas we have limited the counterions from coming within about $2.4 \AA$ from the surface, further investigation revealed that the inclusion of electrostatic images while decreasing the optimal ion-surface distance to about $0.5 \AA$ can increase the rod-surface equilibrium distance by $1 \AA$ compared to the case without electrostatic images. Thus, the inclusion of electrostatic images in these systems seems to introduce only rather short-ranged, repulsive effects. This raises a question for real aqueous systems and their modeling: To what extent do the hydration layers around macroions conceal electrostatic image effects?

There are many variations of this system that one could explore. For example, in the present system the effective rod-surface force could be studied as a function of the ratio of monovalent and divalent counterions. As the strengths of the electrostatic interactions involving monovalent counterions would be less than those involving divalent counterions, the "dilution" of a divalent-counterion system by the addition of monovalent counterions would be expected to decrease the attractive force between the rod and surface. Another variation 
of the present system geometry that has immediate relevance to experiments is system consisting of oppositely charged rods and surface. In particular, Yang and Fang66 imaged DNA strands adsorbed onto a supported cationic lipid bilayer using atomic force microscopy

and found that the DNA spacing in the fingerprint-like images increased monotonically from about 40 to $60 \AA$ as the sodium chloride concentration increased from about $20 \mathrm{mM}$ to $1 \mathrm{M}$. This latter subject will be addressed in a future article.

\section{ACKNOWLEDGMENTS}

We thank Robijn Bruinsma, William Gelbart, and Philip Pincus for stimulating discussions. This work was performed under the auspices of the United States Department of Energy and was supported in part by NSF grant DMR-9708646. 


\section{REFERENCES}

${ }^{1}$ L. Guldbrand, B. Jönsson, H. Wennerström, and P. Linse. J. Chem. Phys. 80, 2221 (1984).

${ }^{2}$ B. Svensson and B. Jönsson, Chem. Phys. Lett. 108, 580 (1984).

${ }^{3}$ D. Bratko, B. Jönsson, and H. Wennerström, Chem. Phys. Lett. 128, 449 (1986).

${ }^{4}$ L. Guldbrand, L. G. Nilsson, and L. Nordenskiöld, J. Chem. Phys. 85, 6686 (1986); L. G. Nilsson, L. Guldbrand, and L. Nordenskiöld, Mol. Phys. 72, 177 (1991).

5 T. Åkesson, C. E. Woodward, B. Jönsson, Phys. Scr. T33, 32 (1990).

${ }^{6}$ J. P. Valleau, R. Ivkov, and G. M. Torrie, J. Chem. Phys. 95, 520 (1991).

${ }^{7}$ R. Kjellander, T. Åkesson, B. Jönsson, and S. Marčelja, J. Chem. Phys. 97, 1424 (1992).

${ }^{8}$ P. B. Bolhuis, T. Åkesson, and B. Jönsson, J. Chem. Phys. 98, 8096 (1993).

${ }^{9}$ A. P. Lyubartsev and L. Nordenskiöld, J. Phys. Chem. 99, 10373 (1995).

${ }^{10}$ N. Grønbech-Jensen, R. J. Mashl, R. F. Bruinsma, and W. M. Gelbart, Phys. Rev. Lett. 78, 2477 (1997).

${ }^{11}$ R. Podgornik, Chem. Phys. Lett. 174, 191 (1990).

${ }^{12}$ F. Oosawa, Biopolymers 6, 134 (1968); Polyelectrolytes, (Marcel Dekker, New York, 1971).

${ }^{13}$ M. Medina-Noyola and D. A. McQuarrie, J. Chem. Phys. 73, 6279 (1980).

${ }^{14}$ G. N. Patey, J. Chem. Phys. 72, 5763 (1980).

${ }^{15}$ R. Kjellander and S. Marčelja, Chem. Phys. Lett. 112, 49 (1984); 114, 124(E) (1985).

${ }^{16}$ R. Kjellander and S. Marčelja, J. Chem. Phys. 82, 2122 (1985); R. Kjellander, J. Chem. Phys. 88, 7129 (1988); Ibid. 89, 7649(E) (1988).

${ }^{17}$ R. Kjellander and S. Marčelja, Chem. Phys. Lett. 127, 402 (1986).

18 R. Kjellander and S. Marčelja, J. Phys. Chem. 90, 1230 (1986).

${ }^{19}$ M. Lozada-Cassou and D. Henderson, Chem. Phys. Lett. 127, 392 (1986).

${ }^{20}$ R. Kjellander, S. Marčelja, and J. P. Quirk, J. Coll. Int. Sci. 126 194, (1988).

${ }^{21}$ R. Kjellander and S. Marčelja, J. Phys. (France) 49, 1009 (1988).

${ }^{22}$ R. Kjellander, S. Marčelja, R. M. Pashley, and J. P. Quirk, J. Phys. Chem. 92, 6489 (1988); J. Chem. Phys. 92, 4399 (1990); R. M. Pashley and J. N. Israelachvili, J. Coll. Int. Sci. 101, 511 (1984).

23 S. E. Feller and D. A. McQuarrie, Mol. Phys. 80, 721 (1993).

24 J. A. Greathouse and D. A. McQuarrie, J. Coll. Int. Sci. 181, 319 (1996).

25 S. Marčelja, Biophys. J. 61, 1117 (1992).

${ }^{26}$ P. Attard and S. J. Miklavic, J. Chem. Phys. 99, 6078 (1993).

${ }^{27}$ M. J. Stevens and M. O. Robbins, Europhys. Lett. 12, 81 (1990). 
${ }^{28}$ Z. Tang, L. E. Scriven, and H. T. Davis, J. Chem. Phys. 97, 9258 (1992).

${ }^{29}$ J. Ray and G. S. Manning, Langmuir 10, 2450 (1994).

${ }^{30}$ I. Rouzina and V. A. Bloomfield, J. Phys. Chem. 100, 9977 (1996).

${ }^{31}$ J.-L. Barrat and J.-F. Joanny, Adv. Chem. Phys. 94, 1 (1996).

${ }^{32}$ V. A. Bloomfield, Biopolymers 31, 1471 (1991).

${ }^{33}$ V. A. Bloomfield, Curr. Op. Struct. Biol. 6, 334 (1996), and references therein.

${ }^{34}$ N. Ise, T. Okubo, K. Yamamoto, H. Kawai, T. Hashimoto, M. Fujimura, and Y. Hiragi, J. Am. Chem. Soc. 102, 7901 (1980).

${ }^{35}$ N. Ise, Angew. Chem. 25, 323 (1986), and references therein.

${ }^{36}$ N. Ise, Ber. Bunsenges. Phys. Chem. 100, 841 (1996), and references therein.

${ }^{37}$ D. C. Rau and V. A. Parsegian, Biophys. J. 61, 246 (1992).

${ }^{38}$ D. C. Rau and V. A. Parsegian, Biophys. J. 61, 260 (1992).

${ }^{39}$ M. Sedlák and E. J. Amis, J. Chem. Phys. 96, 817 (1992).

${ }^{40}$ M. Sedlák and E. J. Amis, J. Chem. Phys. 96, 826 (1992).

${ }^{41}$ J. X. Tang, S. Wong, P. T. Tran, P. A. Janmey, Ber. Bunsenges. Phys. Chem. 100, 796 (1996).

${ }^{42}$ A. Khan, K. Fontell, and B. Lindman, J. Coll. Int. Sci. 101, 193 (1984); A. Khan, B. Jönsson, and H. Wennerström, J. Phys. Chem. 89, 5180 (1985).

${ }^{43}$ P. G. Slade and J. P. Quirk, J. Coll. Int. Sci. 144 18, (1991).

${ }^{44}$ G. Gouy, J. Phys. (France) 9, 457 (1910); Ann. Phys. 7, 129 (1917); D. L. Chapman, Philos. Mag. 25, 475 (1913).

${ }^{45}$ S. L. Brenner and V. A. Parsegian, Biophys. J. 14, 327 (1974).

${ }^{46}$ S. L. Brenner, V. A. Pargsegian, and D. Gingell, J. Phys. Chem. 82, 1727 (1978).

${ }^{47}$ S. L. Carnie, D. Y. C. Chan, and J. Stankovich, J. Coll. Int. Sci. 165, 116 (1994).

${ }^{48}$ S. Y. Shulepov and Y. V. Shulepov, J. Coll. Int. Sci. 172, 185 (1995).

${ }^{49}$ J. G. Kirkwood, J. Chem. Phys. 2, 767 (1934).

${ }^{50}$ H. G. Hansma HG, R. L. Sinsheimer, J. Groppe, T. C. Bruice, V. Elings, G. Gurley, M. Bezanilla, I. A. Mastrangelo, P. V. Hough, P. K. Hansma, Scanning 15, 296 (1993).

${ }^{51}$ Y. L. Lyubchenko, B. L. Jacobs, S. M. Lindsay, A. Stasiak, Scanning Microsc. 9, 705 (1995).

${ }^{52}$ C. Bustamante, J. Vesenka, C. L. Tang, W. Rees, M. Guthold, and R. Keller, Biochemistry 31, 22 (1992).

${ }^{53}$ H. G. Hansma, R. L. Sinsheimer, M. Q. Li, P. K. Hansma, Nucleic Acids Res. 20, 3585 (1992). 
${ }^{54}$ H. G. Hansma, J. Vesenka, C. Siegerist, G. Kelderman, H. Morrett, R. L. Sinsheimer, V. Elings, C. Bustamante, and P. K. Hansma, Science 256, 1180 (1992).

${ }^{55}$ E. Henderson, Nucleic Acids Res. 20, 445 (1992).

${ }^{56}$ J. Vesenka, M. Guthold, C. L. Tang, D. Keller, E. Delaine, and C. Bustamante, Ultramicroscopy 42-44, 1243 (1992).

${ }^{57}$ H. G. Hansma, H. Bezanilla, F. Zenhausern, M. Adrian, and R. L. Sinsheimer, Nucleic Acids Res. 21, 505 (1993).

${ }^{58}$ M. Guthold, M. Bezanilla, D. A. Erie, B. Jenkins, H. G. Hansma, and C. Bustamante, Proc. Natl. Acad. Sci. USA 91, 12927 (1994).

${ }^{59}$ E. Delain, A. Fourcade, J. C. Poulin, A. Barbin, D. Coulaud, E. Lecam, E. Paris, Microsc. Microanal. Microstruct. 3, 457, (1992).

${ }^{60}$ F. Zenhausern F, M. Adrian, B. ten Heggeler-Bordier, R. Emch, M. Jobin, M. Taborelli, P. Descouts, J. Struct. Biol. 108, 69 (1992).

${ }^{61}$ F. Zenhausern, L. M. Eng, M. Adrian, S. Kasas, A. L. Weisenhorn, and P. Descouts, Helv. Phys. Acta 65, 820 (1992).

${ }^{62}$ A. Shaper, J. P. P. Starink, and T. M. Jovin, FEBS Lett. 355, 91 (1994).

${ }^{63}$ G. Romanowski, M. G. Lorenz, and W. Wackernagel, Appl. and Environ. Microbiology 57, 1057 (1991).

${ }^{64}$ J. E. Hagstrom, M. G. Sebestyen, V. Budker, J. J. Ludtke, J. D. Fritz, and J. A. Wolff, Biochim. Biophys. Acta 1284, 47 (1996).

${ }^{65}$ C.-Y. Wang and L. Huang, Biochem. and Biophys. Res. Comm. 147, 980 (1987).

${ }^{66}$ Y. Fang and J. Yang, J. Phys. Chem. B 101, 441 (1997).

${ }^{67}$ Y. Fang and J. Yang, J. Phys. Chem. B 101, 3453 (1997).

68 J. D. Jackson, Classical Electrodynamics, Second Ed., (Wiley \& Sons, New York, 1975), Ch. 4.

${ }^{69}$ N. Grønbech-Jensen, G. Hummer, and K. M. Beardmore, Mol. Phys. 92, 941 (1997).

${ }^{70}$ N. Grønbech-Jensen, Int. J. of Mod. Phys. C. 7, 873, (1996); Ibid. 8, 1287 (1997).

${ }^{71}$ L. Stryer, Biochemistry, Fourth ed. (Freeman and Company, New York, 1995), Ch. 31.

${ }^{72}$ M. J. Rosen, Surfactants and Interfacial Phenomena, (Wiley \& Sons, New York, 1989).

${ }^{73}$ F. Livolant, A. M. Levelut, J. Doucet, and J. P. Benoit, Nature 339, 724 (1989).

${ }^{74}$ R. Podgornik, H. H. Strey, D. C. Rau, and V. A. Parsegian, Biophys. Chem. 57, 111 (1995).

${ }^{75}$ A. P. Lyubartsev and A. Laaksonen, J. Phys. Chem. 100, 16410 (1996).

${ }^{76}$ M. P. Allen and D. J. Tildesley, Computer Simulation of Liquids, (Oxford, New York, 1987).

${ }^{77}$ D. L. Ermak and Y. Yeh, Chem. Phys. Lett. 24, 243 (1974). 
${ }^{78}$ D. L. Ermak, J. Chem. Phys. 62, 4189 (1975).

${ }^{79}$ Y. Xu and F. C. Szoka, Jr., Biochem. 35, 5616 (1996). 


\section{FIGURES}

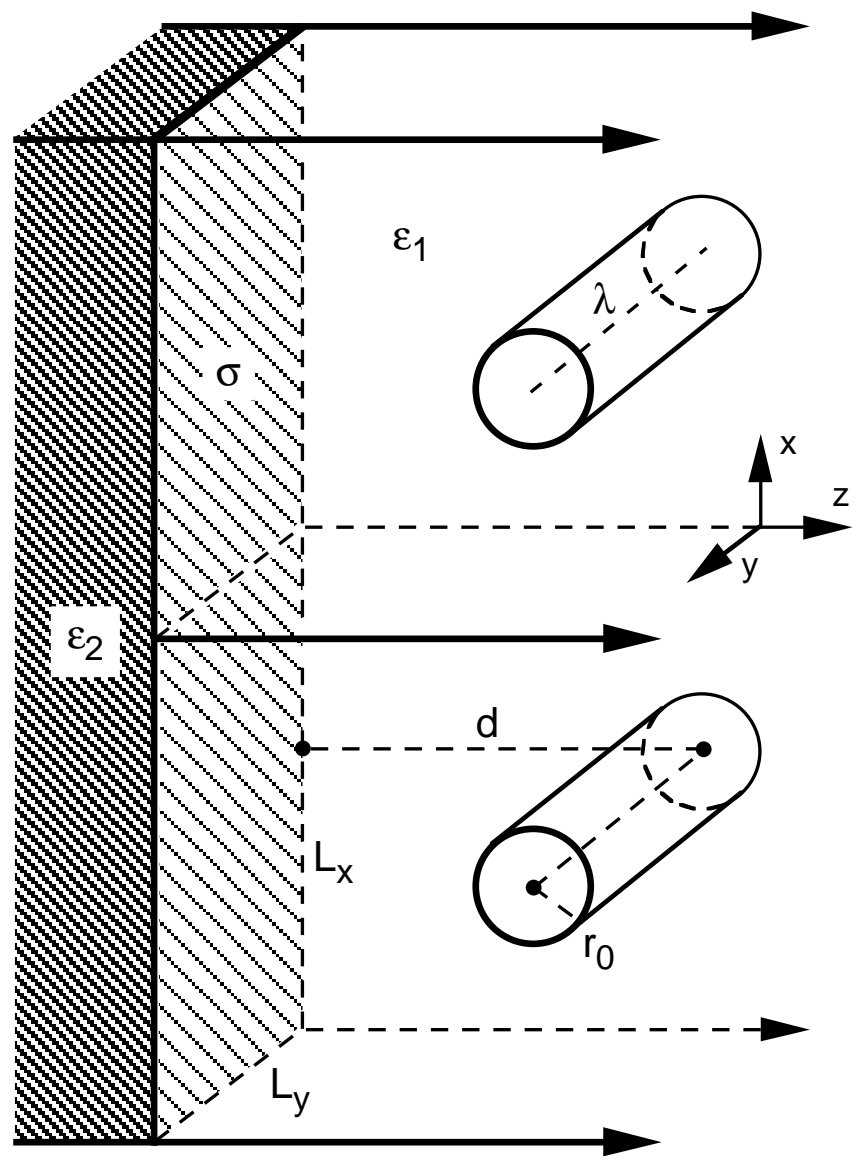

FIG. 1. The simulation system as described in the text. Periodic boundary conditions applied to a unit cell of lateral dimensions $L_{x}$ and $L_{y}$ produce an infinite array of infinitely long line charges with a spacing of $L_{x}$. Each line charge with uniform charge density $\lambda$ and radius $r_{0}$ is located in an isotropic, continuous medium of dielectric constant $\epsilon_{1}$ and positioned a distance $d$ from a charged surface at $z=0$ with average density $\sigma$. The replicated $\epsilon_{1}$ medium is bounded by a second isotropic, continuous medium of dielectric constant $\epsilon_{2}$. 


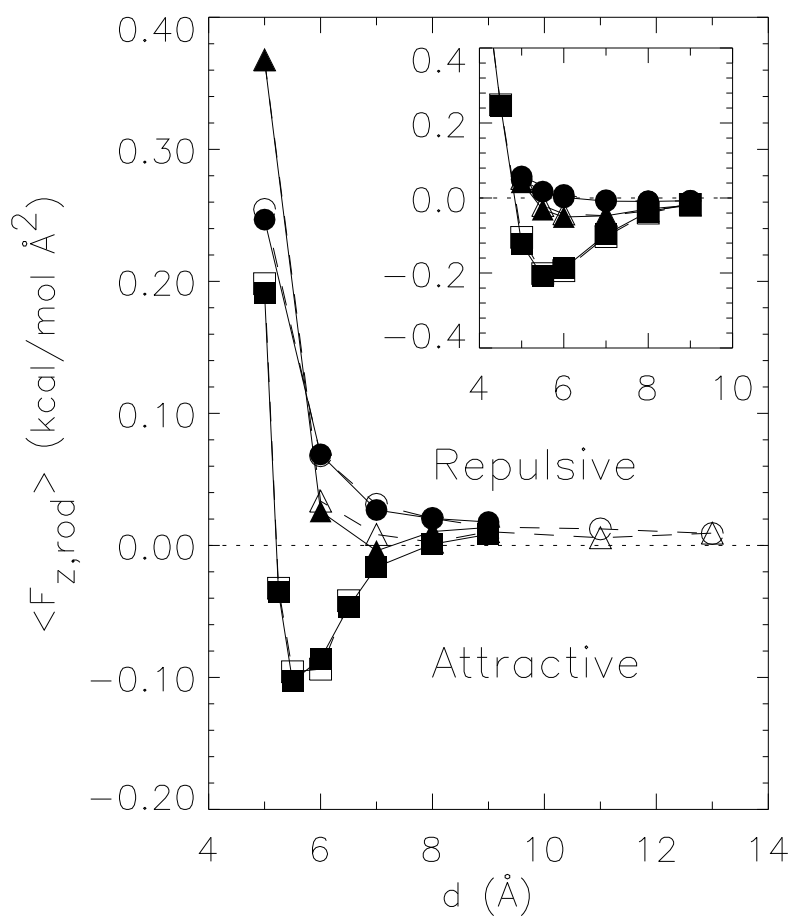

FIG. 2. Force-distance profiles for the effective, timeaveraged force on the rod (per unit length) perpendicular to the surface for a given fixed rod-surface distance $d$. Shown are a series of curves for a system of monovalent counterions with (dashed lines, open symbols) and without (solid lines, filled symbols) electrostatic images. The symbols correspond to the following uniform charge densities: $(\bullet, \circ) \lambda=\lambda_{\text {ref }}, \sigma=\sigma_{\text {ref }} ;(\boldsymbol{\Lambda}, \Delta) \lambda=2 \lambda_{\text {ref }}, \sigma=2 \sigma_{\text {ref }} ;(\boldsymbol{\square}, \square) \lambda=4 \lambda_{\text {ref }}, \sigma=4 \sigma_{\text {ref }}$. The inset shows the analogous series for a system of divalent counterions with the following charge densities: $(\bullet, \circ) \lambda=0.5 \lambda_{\text {ref }}, \sigma=0.5 \sigma_{\text {ref }} ;(\boldsymbol{\Lambda}, \Delta) \lambda=\lambda_{\text {ref }}, \sigma=\sigma_{\text {ref }} ;(\boldsymbol{\square}, \square) \lambda=2 \lambda_{\text {ref }}, \sigma=2 \sigma_{\text {ref }}$. The sizes of the error bars do not exceed those of the figure symbols and are not shown. In all cases, $L_{x}=30 \AA, r_{0} \approx 2.8 \AA, \lambda_{\text {ref }}=-e / 1.7 \AA$, and $\sigma_{\text {ref }}=-e / 51 \AA^{2}$. 


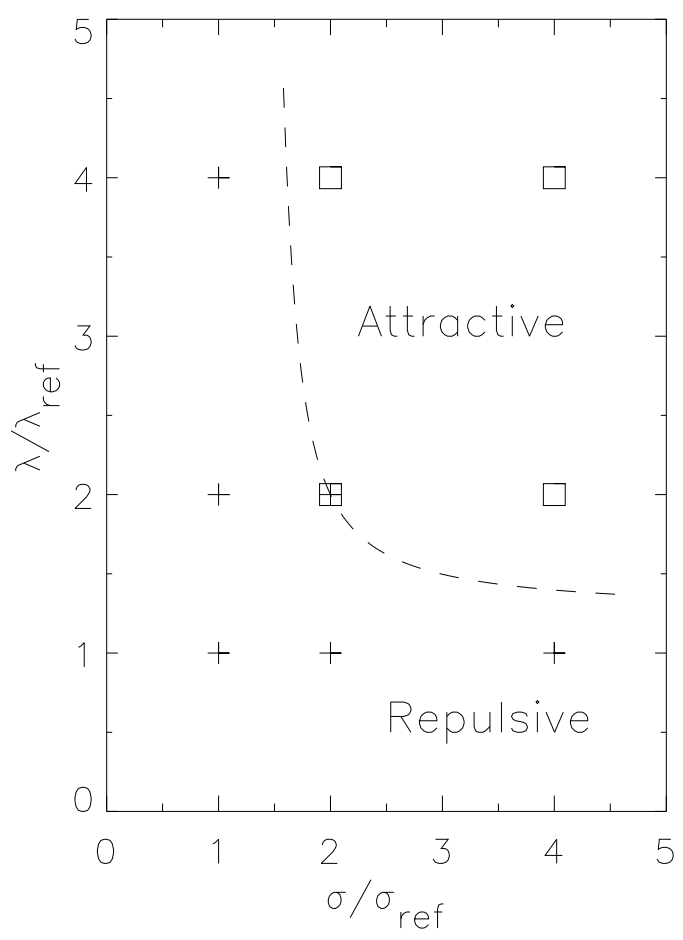

FIG. 3. Phase diagram for a system of monovalent counterions, indicating regions in which the rod-surface interaction is attractive $(\square)$ and repulsive $(+)$ for several values of uniform rod linear charge densities $\lambda$ and uniform surface charge densities $\sigma$, relative to the reference system with $\lambda_{\text {ref }}$ and $\sigma_{\text {ref. }}$. The point at $\lambda / \lambda_{\text {ref }}=\sigma / \sigma_{\text {ref }}=2$ corresponds to a system that shows only marginal attraction (see Fig. 2) and is marked to indicate that it is a boundary point. The dashed line indicates qualitatively the boundary between the regions. In all cases, $L_{x}=30 \AA, r_{0} \approx 2.8 \AA$, $\lambda_{\text {ref }}=-e / 1.7 \AA$ and $\sigma_{\text {ref }}=-e / 51 \AA^{2}$. 


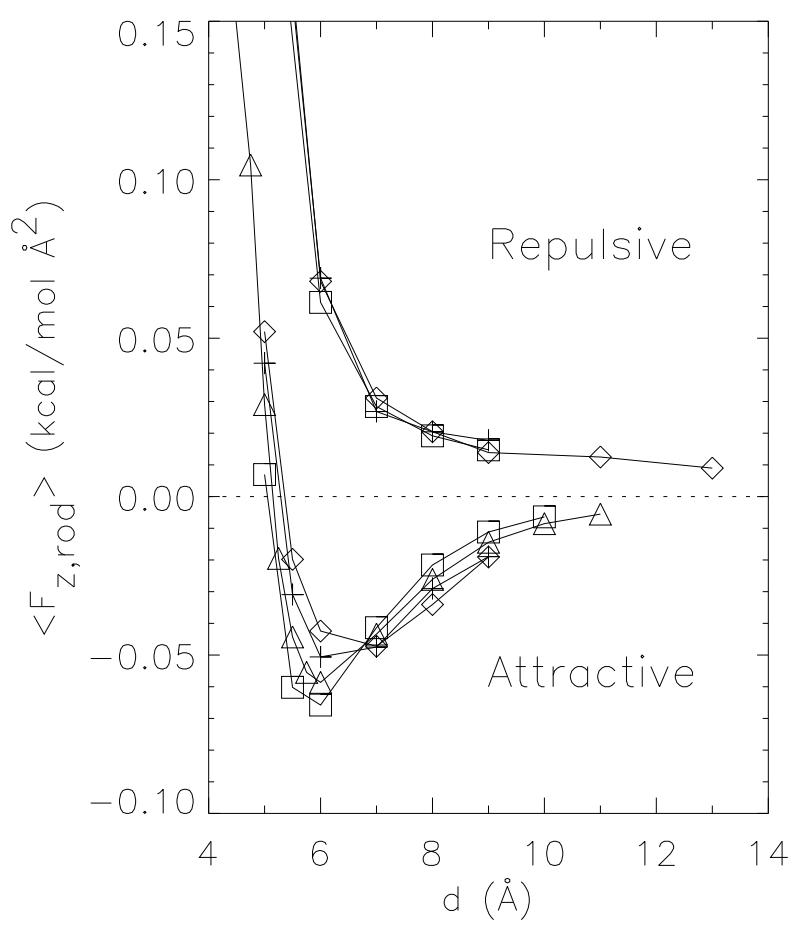

FIG. 4. Comparison of systems with or without electrostatic images, having surfaces with either uniform charge densities or discrete mobile monovalent anionic charge distributions for $\lambda=\lambda_{\text {ref }}$ and $\sigma=\sigma_{\text {ref }}$. The upper curves correspond to systems of monovalent counterions; the lower curves, divalent. The following key applies to both sets of curves: (+) uniform with no electrostatic images, $(\diamond)$ uniform with electrostatic images, $(\triangle)$ discrete mobile surface with no electrostatic images, $(\square)$ discrete mobile surface with electrostatic images. The sizes of the error bars do not exceed those of the figure symbols and are not shown. In all cases, $L_{x}=30 \AA, r_{0} \approx 2.8 \AA, \lambda_{\text {ref }}=-e / 1.7 \AA$ and $\sigma_{\text {ref }}=-e / 51 \AA^{2}$. 